\title{
Ultrafast two-dimensional nuclear magnetic resonance spectroscopy of hyperpolarized solutions
}

\author{
LUCIO FRYDMAN ${ }^{*}$ AND DAMIR BLAZINA² \\ ${ }^{1}$ Department of Chemical Physics, Weizmann Institute, Rehovot 76100, Israel \\ ${ }^{2}$ Oxford Instruments Molecular Biotools Ltd, OX13 5QX, UK \\ *e-mail: Lucio.Frydman@weizmann.ac.il
}

Two-dimensional (2D) NMR is an important tool for elucidating molecular structure and dynamics ${ }^{1}$. However, the method is limited by the low sensitivity inherent to NMR techniques, resulting in typical acquisition times for $2 \mathrm{D}$ NMR spectra ranging from minutes to hours. A number of hyperpolarization techniques have been explored to boost NMR's sensitivity, including an ex situ dynamic nuclear polarization method capable of yielding - for an array of molecules and under conventional observation conditions for liquid samplessignals that exceed those currently afforded by the highestfield spectrometers by several orders of magnitude ${ }^{2}$. Whereas this methodology is able to provide the sensitivity equivalent of $\sim 10^{6}$ scans, it is constrained to extract its 'super-spectrum' within a single transient, making it a poor starting point for conventional 2D NMR acquisitions. Here, we show that if the ex situ dynamic nuclear polarization approach is suitably merged with spatially encoded ultrafast NMR spectroscopy ${ }^{3}, 2 D$ NMR spectra of liquid samples at submicromolar concentrations can be acquired within $\sim 0.1 \mathrm{~s}$.

The future of NMR hinges on the efforts devoted to increase the resolution and sensitivity of this branch of spectroscopy. Key among the physical concepts that define NMR's resolving power are multidimensional $(n \mathrm{D})$ acquisitions, which spread and correlate the resonances arising from individual sites onto multiple frequency axes ${ }^{1,4,5}$. Although these experiments are intrinsically lengthier than their conventional one-dimensional (1D) counterparts, $n \mathrm{D}$ NMR's success is best portrayed by its nearly ubiquitous contemporary practice in chemistry and biology, as well as by its various NMR imaging (MRI) clinical derivations. Comparable improvements regarding NMR's sensitivity, a particularly pressing issue given the weak signals associated with magnetic resonance observations in bulk, have been slower in coming. Moreover, the moderate dependence that sensitivity exhibits on the magnetic field, $B_{\mathrm{NMR}}$, has led to diminishing returns despite investments on ever-larger NMR magnets. Driven by this reality, recently there has been an increased interest in devising alternatives that prepare nuclei in 'hyperpolarized' states, whose spin population differences depart from the usual $\approx 10^{-5}$ Boltzmann distributions and approach unity values. Methods proposed and demonstrated for achieving such metastable spin states include chemical synthesis and parahydrogen ${ }^{6,7}$, optical pumping ${ }^{8,9}$ and microwave-driven transfers of magnetization from electrons to nearby nuclei via dynamic nuclear polarization ${ }^{10-13}$ (DNP). DNP is arguably the most generally applicable of these methods, providing relatively high enhancements reaching up to the $\gamma_{\text {electron }} / \gamma_{\text {nucleus }}$ ratio between the spins' magnetogyric constants, while having the relatively modest requirement that the targeted system be mixed with a free radical to be irradiated at its Larmor frequency.

A logical approach to exploit the benefits of DNP within a high-resolution liquid-state NMR setting would be to carry out the microwave irradiation in situ, at high fields and in the phase where the sample is to be studied. However, the inefficiency of electron-nuclear spin transfer mechanisms in liquids at high fields, compounded by the relatively short relaxation times of these species in solution, makes this type of operation impractical ${ }^{11-13}$. As shown by Griffin et al. with the aid of custom-built microwave and solid-state NMR instrumentation, many of these problems can be dealt with by carrying out the in situ polarizing procedure at low temperatures on frozen glassy samples ${ }^{14,15}$, a proposition recently extended to liquids by following it up with a laseraided temperature jump ${ }^{16}$. In a related approach geared towards liquid-phase NMR and MRI observations, Golman and co-workers proposed carrying out the DNP ex situ from the NMR magnet, and rely on a rapid melting and transfer of the hyperpolarized sample into the scanner for an otherwise routine NMR/MRI observation $^{2,17}$. The relatively long microwave irradiation times needed by this ex situ DNP procedure were supported by operating at cryogenic temperatures, where nuclear spin polarizations could be allowed to build up over hours. Notwithstanding the relaxation losses involved in the subsequent melting and transfer of the sample into the NMR magnet, a significantly hyperpolarized liquid still results, factoring in both the DNP and the sudden melting contributions as $\left(\gamma_{\text {electron }} / \gamma_{\text {nucleus }}\right)^{*}\left(B_{\mathrm{DNP}} T_{\mathrm{NMR}} / B_{\mathrm{NMR}} T_{\mathrm{DNP}}\right)$. Even when accounting for the relatively low field in which DNP takes place $\left(B_{\mathrm{DNP}} / B_{\mathrm{NMR}} \approx 1 / 8\right)$ and the fact that most contemporary NMR experiments nowadays rely on a proton-driven polarization transfer (that is, $\gamma_{\text {electron }} / \gamma_{\text {nucleus }} \approx 665$ ), the involved factors can still deliver enhancements $\geq 15,000$-much larger than what is currently achievable by conventional multiscan signal averaging.

Although carrying a proven potential for improving the sensitivity of high-resolution liquid-state NMR, the ex situ DNP-based procedure just described is also associated with one apparent drawback: as it involves an irreversible melting and 


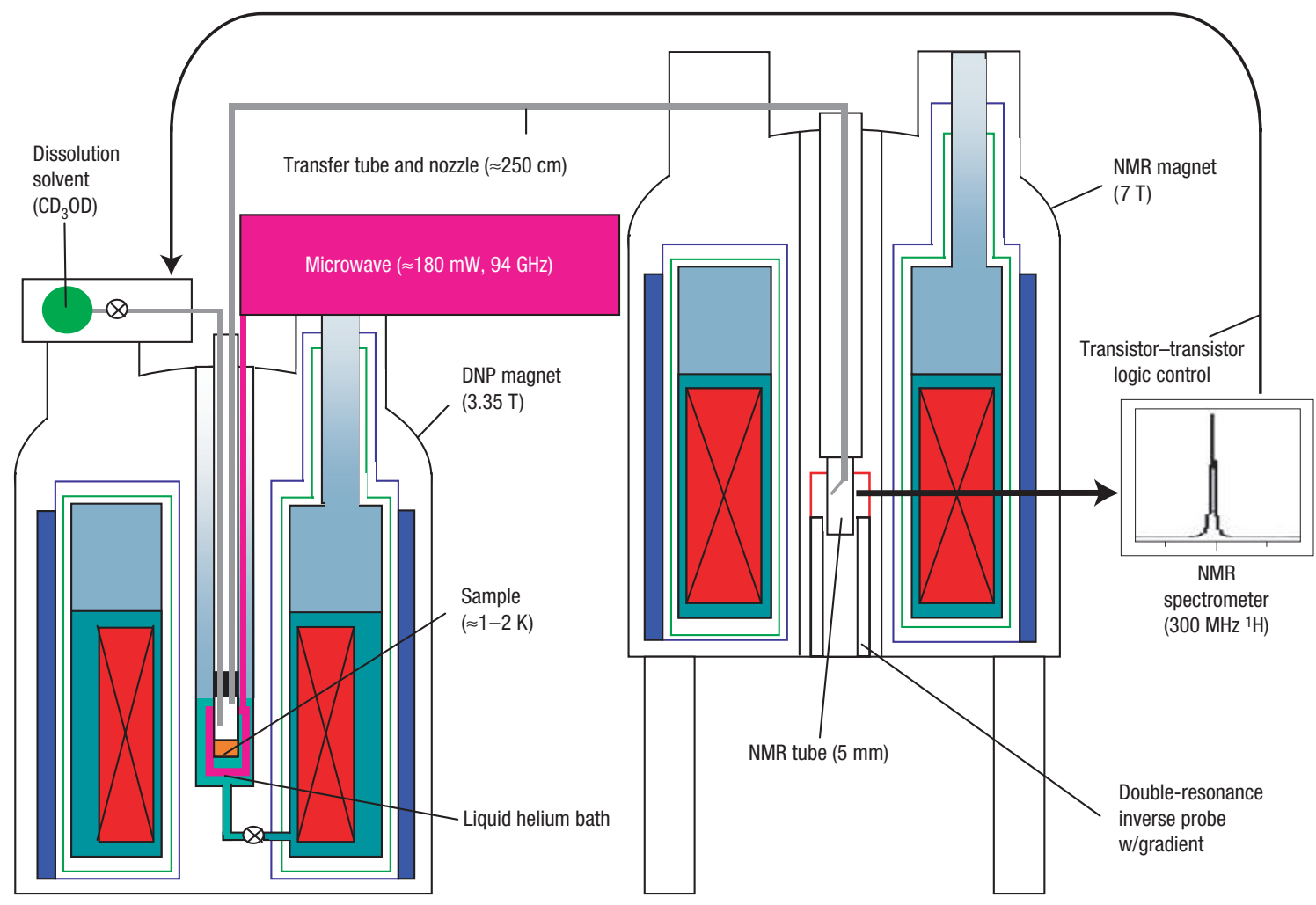

Figure 1 Schematic diagram of the combined DNP/NMR set-up used in this study. The sample to be investigated was initially hyperpolarized in the cold magnet system shown on the left, and subsequently transferred by a stream of hot solvent into the NMR system on the right for its actual observation. Except for the transfer assembly, all of the NMR-related components (tube, probe, console, magnet) are standard; further experimental details are given in the text.

transfer of the sample, it is ill-suited for the acquisition of multiple scans of comparable intensities. This seems to make it incompatible with $n \mathrm{D}$ NMR spectroscopy, which is based on collecting an array of scans that are identical to one another except for the systematic incrementation of certain evolution delays ${ }^{4,5}$. Similar situations were dealt with in the $n \mathrm{D}$ MRI of optically pumped gases by relying on the use of small flip-angle pulses exciting only a fraction of the magnetization within each increment ${ }^{8,18}$; however, the complexity of most $2 \mathrm{D}$ spectroscopic sequences coupled to the fact that transverse and longitudinal relaxation times in liquids are comparable, conspire against adopting similar strategies in high-resolution NMR. To deal with this constraint, in this study we explore the integration of ex situ DNP with the recently proposed ultrafast $n \mathrm{D}$ NMR techniques ${ }^{3}$. We assay in particular an ultrafast approach where the serial indirect domain $t_{1}$ encoding of $2 \mathrm{D}$ NMR is replaced by a parallelized procedure endowing different positions, $z$, within a sample of length, $L$, with inequivalent evolution times, $0 \leq t_{1} \leq t_{1}^{\max }$. This can be carried out with the aid of an imaging-like magnetic-field gradient, $G_{\mathrm{e}}$, acting in combination with a frequency-swept excitation of the spins. Together, these manipulations can create a spin coherence pattern, $\rho(z)=\rho_{0} \exp \left[i C \Omega_{1}\left(z-z_{0}\right)\right]$, defined by a known spatiotemporal ratio, $C \approx t_{1}^{\max } / L$, and by the unknown $\Omega_{1}$ interaction to be measured ${ }^{3,19}$. Such indirect-domain spatial winding is preserved throughout the various manipulations that make up the $2 \mathrm{D}$ mixing period, and finally read out during the acquisition time $t_{2}$ using a second, oscillating field gradient, $G_{\mathrm{a}}$, possessing the same spatial geometry as $G_{\mathrm{e}}$. Whenever the acquisition wavenumber $k \int_{0}^{t_{2}} \gamma_{\mathrm{a}} G_{\mathrm{a}}(t) \mathrm{d} t$ fulfils $k=-C \Omega_{1}$, an echo revealing the nature of the indirect-domain NMR spectrum arises; its Fourier analysis as a function of $t_{2}$ yields a full 2D NMR spectrum identical to that achievable by multiscan means, yet in a single transient.

Owing to its single-scan nature, ultrafast 2D NMR seems to be a useful complement to the rapid-melting ex situ DNP procedure. However, the heavy reliance that ultrafast NMR makes on carefully balanced gradient-based refocusing processes along a spatial axis will make a very poor partnership with the turbulences that the rapid injection underlying the DNP protocol will set in within the NMR tube. To optimize the compatibility between these two procedures, a series of precautions was taken including a choice of solvent (methanol) that minimizes foaming, and a nozzle injection system that introduces the sample onto the side walls of the NMR tube to dampen subsequent turbulences. In the resulting set-up (Fig. 1), a gradient-based spatial encoding procedure could be reliably imparted within $\approx 0.5 \mathrm{~s}$ from the point when the NMR tube was filled with the hyperpolarized solution. This settling time adds to the approximately $2 \mathrm{~s}$ needed for the sudden melting and transfer of the sample from the DNP polarizer to the NMR magnet, leading to an elapse of less than $3 \mathrm{~s}$ between the removal of the sample from its cryogenic hyperpolarizing environment and its measurement in the room-temperature NMR system. Although this delay is too long to accommodate rapidly relaxing ${ }^{1} \mathrm{H}$ nuclei, it was found to be short enough to withstand the transfer losses arising from more slowly relaxing ${ }^{13} \mathrm{C}$ or ${ }^{15} \mathrm{~N}$ heteronuclei. All ultrafast sequences consequently focused on 2D NMR correlations that started from heteronuclear $z$ magnetizations, and transferred the spatially encoded indirect-domain evolution to nearby protons for their direct-domain observation. The resulting inverse-detected experiments are known to maximize NMR sensitivity ${ }^{20-22}$, but also face potential complications arising from strong, undesired 

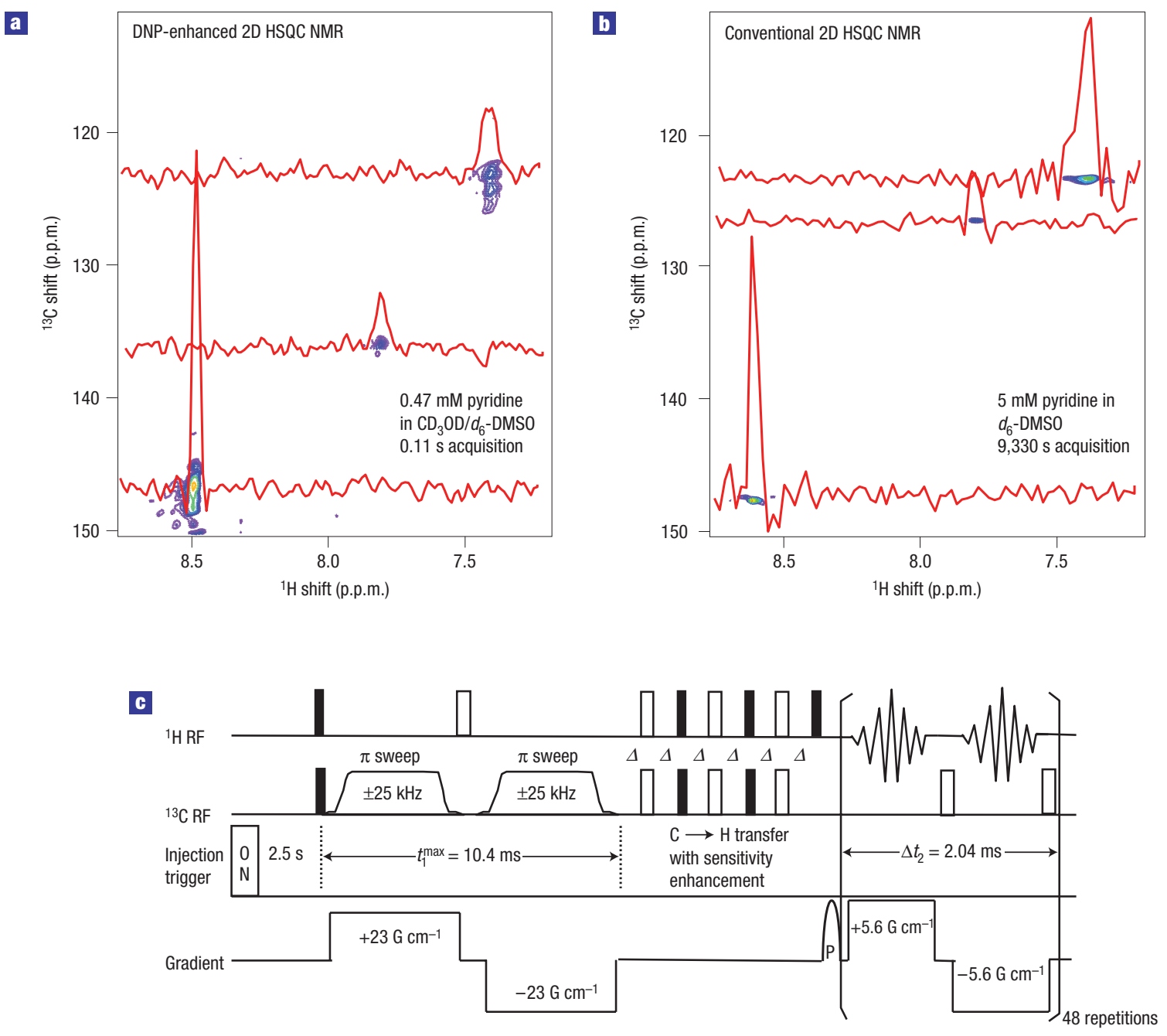

Figure 2 Comparison between DNP-enhanced and conventional 2D HSQC NMR correlations collected on natural-abundance pyridine solutions. a,b, Each part lists the concentrations used and the times elapsed between the first RF pulse and the last experimental data point collected; the cross-sections exhibited by the various peaks in the $2 \mathrm{D}$ experiments are also shown. The conventional spectrum (b) was recorded at a higher field (11.7 versus $7.0 \mathrm{~T}$ ) and in a somewhat different solvent than its DNP counterpart (a); the dissimilar intensities exhibited by peaks in the two spectra reflect the differential build-up and relaxation rates affecting the ex situ hyperpolarization process. c, Single-scan pulse sequence and its acquisition parameters, with full and hollow bars corresponding to $\pi / 2$ and $\pi$ pulses and $\Delta=\left(4 J_{C H}\right)^{-1}$. The conventional spectrum was recorded with analogous timings and spectral widths, yet incorporating an initial ${ }^{1} \mathrm{H} \rightarrow{ }^{13} \mathrm{C}$ transfer before $t_{1}$.

proton background signals. These are usually dealt with by means of multiscan phase-cycling procedures. However, in the present case we observed that trains of spatially encoded acquisitions on hyperpolarized solutions yielded very little signal $(\approx 10 \%)$ past the first transient. Although this observation was encouraging in terms of revealing a very efficient use of the hyperpolarized magnetization ported into the coil region, it also discouraged us from averaging multiple scans. All natural-abundance and solvent background signals were thus overcome by relying solely on the gradientbased suppression of undesired resonances ${ }^{23}$, incorporated into the ultrafast NMR sequences.

Figure 2 compares 2D heteronuclear single-quantum correlation (HSQC) NMR spectra obtained on the basis of these provisions on a hyperpolarized and on a thermally polarized (non-injected) pyridine solution, a sample chosen as a prototype molecule incorporating multiple ${ }^{13} \mathrm{C}-{ }^{1} \mathrm{H}$ correlations. The pulse sequence and further details used in the ultrafast 2D NMR experiment are also shown in the figure. Note that peak positions in the conventional and single-scan 2D spectra are analogous along both dimensions, thus validating the analytical potential of the DNP-enhanced protocol. Sensitivity-wise it seems that the $0.47 \mathrm{mM}$ concentration used in this hyperpolarization test is not much lower than those addressed in previous ultrafast HSQC reports $(\approx 1-3 \mathrm{mM}$ (refs 19,24)) - until it is realized that in contrast to the latter, the ${ }^{13} \mathrm{C}$ isotopes here are being observed at their natural $1 \%$ abundance. When factoring this in, it becomes clear that the ex situ DNP process has improved sensitivity by several orders of magnitude. Ancillary NMR observations reveal that this factor exceeds $10^{3}$, and that the noise appearing in the DNP-enhanced spectrum of Fig. 2 is not of thermal origin but rather a reflection of the residual natural-abundance background arising from ${ }^{1} \mathrm{H}-{ }^{12} \mathrm{C}$ spin pairs. (This background could be substantially reduced by incorporating additional transverse, non-encoding coherenceselection gradient pulses. Unfortunately such hardware was not available during the course of the present project.) These features suggest that considerably lower concentrations than the one 

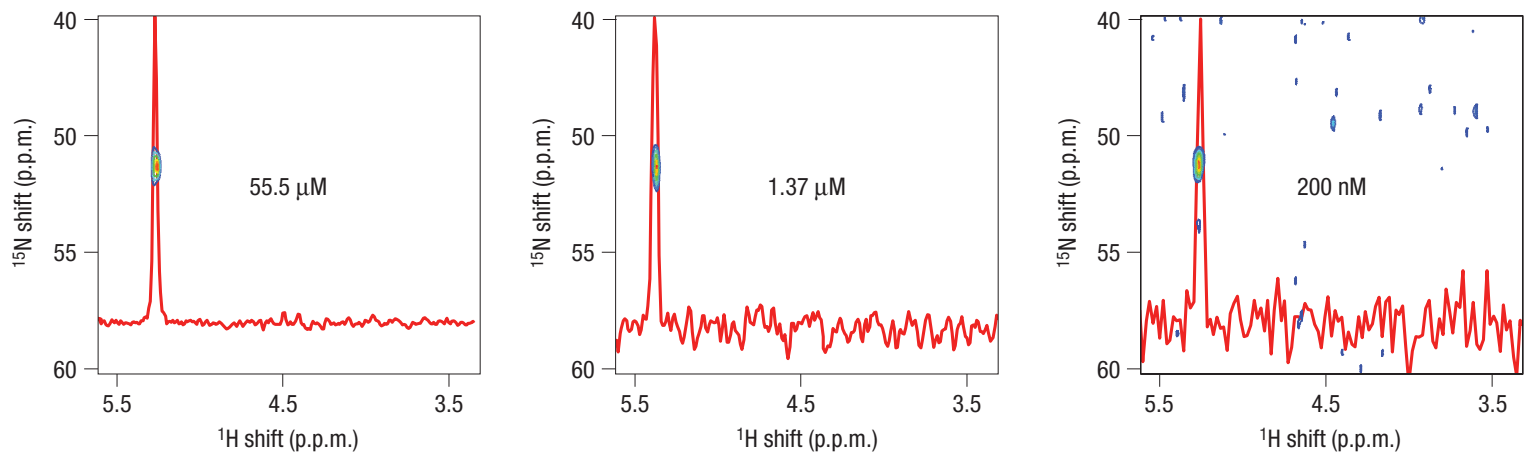

Figure 3 DNP-enhanced single-scan 2D ${ }^{15} \mathrm{~N}-{ }^{1} \mathrm{H}$ HSQC NMR spectra collected on urea solutions at decreasing sample concentrations. Cross-sections taken at the peak's positions to illustrate the variations in spectral sensitivity. All data were collected using a pulse sequence similar to that in Fig. 2 (albeit with no sensitivity enhancement in the $\mathrm{N} \rightarrow \mathrm{H}$ transfer) within approximately $0.13 \mathrm{~s}$ acquisition times.

probed in Fig. 2 should be amenable to study by DNP-enhanced ultrafast 2D NMR, if we were to focus on isotopically labelled systems. To test this hypothesis, a series of HSQC experiments were carried out on ${ }^{15} \mathrm{~N}$-labelled urea, which under the assayed conditions yielded slightly lower ${ }^{15} \mathrm{~N}$ polarizations $(\approx 10 \%)$ than the ${ }^{13} \mathrm{C}$ in $\mathrm{DNP}$-enhanced pyridine $(\approx 20 \%)$. Figure 3 shows a series of single-scan $2 \mathrm{D}{ }^{15} \mathrm{~N}-{ }^{1} \mathrm{H}$ HSQC spectra, recorded on the resulting hyperpolarized solutions at progressively lower amounts of dissolved urea. This series concludes with a urea sample whose concentration is $200 \mathrm{nM}$; a value reflecting our limitations in achieving lower yet well-defined concentrations, rather than an actual noise-based limit of detection for this technique. We believe that the data summarized in Figs 2 and 3 constitute a new frontier in the field of liquid-phase NMR, combining the wealth of information known to characterize multidimensional spectroscopy, an unprecedented sensitivity and remarkably short spectrometer times associated with the information retrieval process-even if factoring in the relatively long times needed by the ex situ DNP pumping process. Indeed, using the sensitivities reported for a usual organic tuning sample in an optimized single-pulse ${ }^{1} \mathrm{H}$ NMR experiment $(0.1 \% \text { ethylbenzene })^{25}$ as a benchmark, it follows that the $\approx 80$ pmol of sample originating the spectrum on the righthand panel of Fig. 3 would require averaging $\geq 10,000$ transients on a $900 \mathrm{MHz}$ NMR spectrometer equipped with a $5 \mathrm{~mm}$ cryogenically cooled probe-head to deliver a comparable signal-to-noise ratio.

By virtue of their unprecedented combination of resolution, speed and sensitivity, the new ultrafast hyperpolarized method discussed here could find immediate applications in a number of frameworks, including studies of small- and medium-sized molecules within the usual settings of chemical, pharmaceutical and metabonomic analyses. Less-traditional research avenues such as studies of solution-state surface phenomena, analyses of transient enzymatic or chemical reaction intermediates, or following in vivo the fate of various metabolites, could also be facilitated by this new type of NMR experiments. Hyperpolarized 2D NMR could also find applications in structural biology, particularly if relying on partial deuteration to reduce the otherwise rapid longitudinal relaxation characterizing proteins and nucleic acids $^{26}$. A particularly encouraging feature in terms of the future prospects of hyperpolarized ultrafast $n \mathrm{D}$ NMR are the numerous improvements that could conceivably be done to extend the sensitivity, resolution and scope of applications of the experiments that were presented here. In terms of the DNP set-up, the sensitivity could be optimized by achieving a more complete polarization of the nuclear spins, whereas the relaxation losses and the quantitativeness deficiencies associated with the ex situ operation could be significantly alleviated by decreasing the time and solvent volumes needed to melt and transfer the sample. From the NMR standpoint, improvements in sensitivity and resolution could also be introduced by operating with more modern instrumentation than the one assayed here, incorporating stronger $B_{\mathrm{NMR}}$ fields, cryogenically cooled probe-heads, transverse gradients encoding additional spectral dimensions, and optimized coil diameters. When considered in unison, all of these steps should suffice to put samples in the $10 \mathrm{nM}$ concentration regime within the reach of numerous ${ }^{1} \mathrm{H}$ - or heteronuclear-based $2 \mathrm{D}$ and $3 \mathrm{D}$ NMR experiments, while preserving the subsecond nature of these spectroscopic acquisitions.

\section{METHODS}

\section{INSTRUMENTAL METHODS}

In a typical DNP run, an aliquot of the sample to be studied together with a radical was inserted in the polarizing magnet at a temperature of $\approx 1.4 \mathrm{~K}$, and irradiated with microwaves at approximately $94 \mathrm{GHz}$. The exact irradiation frequency and power was then fine-tuned for each compound and, after a few hours of irradiation $(\sim 4-6)$, the sample was dissolved by the injection of hot pressurized methanol- $d_{4}(450 \mathrm{~K}, 9 \mathrm{bar})$, which rapidly melted and transferred it into a $5 \mathrm{~mm}$ NMR tube via a polytetrafluoroethylene tubing and nozzle. This NMR tube was located within a commercial spectrometer where the actual measurements took place, under unlocked but otherwise conventional high-resolution conditions. A transistor-transistor logic line from the NMR spectrometer was assigned to initiate the rapid sample melting and transfer process, with typical delays between the triggering of the melting and the start of the NMR pulse sequence of approximately $2.5 \mathrm{~s}$. At the end of this delay, the sample had settled inside the NMR tube at a temperature of approximately $20^{\circ} \mathrm{C}$, and the actual single-scan $2 \mathrm{D}$ pulse sequence was executed. The various parameters required for ultrafast acquisitions (probe-head tuning, radiofrequency offsets and amplitudes, gradient strengths, shimming conditions and so on) were calibrated before the transfer using standard samples and procedures; the only significant distortion observed in these pre-calibrated parameters on carrying out the sudden DNP transfer consisted of an approximately 0.1 p.p.m. shift, which we attribute to operating without a deuterium lock. The type of pulse sequences used throughout the single-scan 2D acquisitions are illustrated in Fig. 2 by a representative example. These sequences differed from published ultrafast HSQC correlations by relying on a direct excitation of the $\mathrm{X}$ heteronuclei; following their spatial encoding these coherences were converted via $\mathrm{X}-{ }^{1} \mathrm{H}$ antiphase states onto ${ }^{1} \mathrm{H}$ signals, purged of background contamination with a short gradient pulse $(\mathrm{P})$, and observed while applying a train of oscillating gradients with synchronized X-spin decoupling. A constant-time version on the basis of a pair of adiabatic $\pi$ sweeps was used for the sake of preserving both quadrature components of the antiphase 
coherences $^{27}$, which could be subsequently transformed into observable signal with a sensitivity enhancement advantage ${ }^{28}$. An initial ${ }^{1} \mathrm{H} \pi / 2$ pulse was also included that exploited the encoding gradients for the sake of better suppressing all of the non-relevant proton background signals. Once collected, the single-scan data were transferred offline and processed as described in the literature (data rearrangement, zero-filling, weighting, 1D Fourier transform and phasing ${ }^{19}$ ) to retrieve the purely absorptive 2D NMR spectra shown in Figs 2 and 3.

\section{SAMPLE PREPARATION}

The pyridine sample used in the DNP test shown in Fig. 2 was prepared by dissolving $1.9 \mathrm{mg}$ of the trityl radical (Tris $\{8$-carboxyl-2,2,6,6-tetra[2-(1hydroxyethyl)]-benzo(1,2-d:4,5-d')bis(1,3)dithiole-4-yl\}methyl sodium salt ${ }^{29}$ ) in a $100 \mu \mathrm{l}$ mixture of natural-abundance pyridine:dimethylsulphoxide (DMSO)- $d_{6}(1: 1)$. The experiment used a $0.15 \mu \mathrm{l}$ aliquot of this solution, which following its DNP hyperpolarization was transferred using $4 \mathrm{ml}$ of methanol- $d_{4}$ to give a final pyridine concentration of $470 \mu \mathrm{M}$ in the NMR tube. The conventional spectrum shown in Fig. 2 was collected using DMSO- $d_{6}$ and a higher pyridine concentration. The urea samples explored in Fig. 3 were prepared from a stock solution containing $0.2 \mathrm{mg}$ of ${ }^{15} \mathrm{~N}_{2}$-urea and $4.4 \mathrm{mg}$ of the trityl radical dissolved in $200 \mu \mathrm{l}$ of a mixture of $\mathrm{D}_{2} \mathrm{O}: \mathrm{DMSO}-d_{6}(1: 1)$. The hyperpolarization tests used different aliquots of this solution, and subsequently diluted them with deuterated methanol during sample transfer. For instance, the most dilute example in Fig. 3 used $0.05 \mu l$ of the urea/trityl stock solution, and dissolved it using $4 \mathrm{ml}$ of methanol- $d_{4}$ to give a final urea concentration of $\sim 200 \mathrm{nM}$.

\section{Received 5 December 2006; accepted 22 March 2007; published 22 April 2007.}

\section{References}

1. Ernst, R. R., Bodenhausen, G. \& Wokaun, A. Principles of Nuclear Magnetic Resonance in One and Two Dimensions (Clarendon, Oxford, 1987).

2. Ardenkjaer-Larsen, J. H. et al. Increase in signal-to-noise ratio of $>10,000$ times in liquid-state NMR Proc. Natl Acad. Sci. USA 100, 10158-10163 (2003).

3. Frydman, L., Scherf, T. \& Lupulescu, A. The acquisition of multidimensional NMR spectra within a single scan. Proc. Natl Acad. Sci. USA 99, 15858-15862 (2002).

4. Jeener, J. in Ampere International Summer School II (Basko Polje, Yugoslavia, 1971).

5. Aue, W. P., Bartholdi, E. \& Ernst, R. R. Two dimensional spectroscopy. Application to nuclear magnetic resonance. J. Chem. Phys. 64, 2229-2246 (1976).

6. Bowers, C. R. \& Weitekamp, D. P. Transformation of symmetrization order in nuclear spin magnetization by chemical reaction and nuclear magnetic resonance. Phys. Rev. Lett. 57, 2645-2648 (1986)

7. Eisenschmid, T. C. et al. Parahydrogen induced polarization in hydrogenation reactions. J. Am. Chem. Soc. 109, 8089-8091 (1987).
8. Albert, M. S. et al. Biological magnetic resonance imaging using laser-polarized ${ }^{129}$ Xe. Nature 370, 199-201 (1994)

9. Navon, G. et al. Enhancement of solution NMR and MRI with laser-polarized xenon. Science 271, 1848-1851 (1996).

10. Carver, T. R. \& Slichter, C. P. Polarization of nuclear spins in metals. Phys. Rev. 92, 212-213 (1953).

11. Hausser, K. H. \& Stehlik, D. Dynamic nuclear polarization in liquids. Adv. Magn. Reson. 3 79-139 (1968)

12. Abragam, A. \& Goldman, M. Nuclear Magnetism: Order and Disorder (Oxford Univ. Press, Oxford, 1982).

13. Müller-Warmuth, W. \& Meise-Gresch, K. Molecular motions and interactions as studied by dynamic nuclear polarization (DNP) in free radical solutions. Adv. Magn. Reson. 11, 1-45 (1983).

14. Becerra, L. R. et al. A Spectrometer for dynamic nuclear polarization and electron paramagnetic resonance at high frequencies. J. Magn. Reson. A 117, 28-40 (1995).

15. Hall, D. A. et al. Polarization-enhanced NMR spectroscopy of biomolecules in frozen solution. Science 276, 930-932 (1997).

16. Joo, C.-G., Hu, K.-N., Bryant, J. A. \& Griffin, R. G. In situ temperature jump high-frequency dynamic nuclear polarization experiments: Enhanced sensitivity in liquid-state NMR spectroscopy. J. Am. Chem. Soc. 128, 9428-9432 (2006).

17. Wolber, J. et al. Generating highly polarized nuclear spins in solution using dynamic nuclear polarisation. Nucl. Instrum. Methods Phys. Res. A 526, 173-181 (2004).

18. Middleton, H. et al. MR imaging with hyperpolarized ${ }^{3} \mathrm{He}$ gas. Magn. Reson. Med. 33, 271-275 (1995).

19. Frydman, L., Scherf, T. \& Lupulescu, A. Principles and features of single-scan two-dimensional NMR spectroscopy. J. Am. Chem. Soc. 125, 9204-9217 (2003).

20. Maudsley, A. A. \& Ernst, R. R. Indirect detection of magnetic resonance by heteronuclear two-dimensional spectroscopy. Chem. Phys. Lett. 50, 368-372 (1977).

21. Müller, L. Sensitivity enhanced detection of weak nuclei using heteronuclear multiple quantum coherence. J. Am. Chem. Soc. 101, 4481-4484 (1979).

22. Bodenhausen, G. \& Ruben, D. J. Natural abundance ${ }^{15} \mathrm{~N}$ NMR by enhanced heteronuclear spectroscopy. Chem. Phys. Lett. 69, 185-189 (1980).

23. Vuister, G. W. et al. Gradient-enhanced HMQC and HSQC spectroscopy. Applications to ${ }^{15} \mathrm{~N}$-labeled Mnt repressor. J. Am. Chem. Soc. 113, 9688-9690 (1991).

24. Gal, M., Mishkovsky, M. \& Frydman, L. Real-time monitoring of chemical transformations by ultrafast 2D NMR spectroscopy. J. Am. Chem. Soc. 128, 951-956 (2006).

25. Kovacs, H., Moskau, D. \& Spraul, M. Cryogenically cooled probes-A leap in NMR technology. Progr. Nucl. Magn. Reson. Spectrosc. 46, 131-155 (2005).

26. Gardner, K. H. \& Kay, L. E. The use of ${ }^{2} \mathrm{H},{ }^{13} \mathrm{C},{ }^{15} \mathrm{~N}$ multidimensional NMR to study the structure and dynamics of proteins. Ann. Rev. Biophys. Biomol. Struct. 27, 357-406 (1998).

27. Pelupessy, P. Adiabatic single-scan 2D NMR spectroscopy. J. Am. Chem. Soc. 125 $12345-12350$ (2003)

28. Kay, L. E., Keifer, P. \& Saarinen, T. Pure absorption gradient enhanced heteronuclear single quantum correlation spectroscopy with improved sensitivity. J. Am. Chem. Soc. 114, 10663-10665 (1992).

29. Thaning, M. Free radicals. US patent 6,013,810 (2000).

\section{Acknowledgements}

We are grateful to M. Gal (WIS) as well as to M. vanCriekinge, A. Sowerby and S. Reynolds (OIMBL) for assistance and support during the course of this project. This work was supported by the US-Israel Binational Science Foundation (BSF 2004298).

Correspondence and requests for materials should be addressed to L.F.

Competing financial interests

The authors declare no competing financial interests.

Reprints and permission information is available online at http://npg.nature.com/reprintsandpermissions/ 